\section{PERCEPÇÃO DOS PAIS SOBRE A IMPLANTAÇÃO DA ESTRATÉGIA DE FORTIFICAÇÃO DAALIMENTAÇÃO INFANTIL COM MICRONUTRIENTES}

\author{
Parents' perception of the implementation of a strategy for the \\ fortification of child food with micronutrients
Percepción de los padres sobre la implantación de la estrategia de fortificación de la alimentación infantil con micronutrientes

Artigo Original

\section{RESUMO}

Objetivo: Analisar a percepção dos pais dos educandos acerca da implantação da estratégia de fortificação da alimentação infantil com micronutrientes em pó (NutriSUS). Métodos: Pesquisa qualitativa, descritiva e exploratória, realizada nos meses de agosto e setembro de 2015, com 11 mães de alunos matriculados nos Centros de Educação Infantil da cidade de Montes Claros, MG, Brasil. Os dados foram coletados por meio de uma entrevista semiestruturada, sendo analisados pela técnica de análise de conteúdo, sendo identificadas duas categorias temáticas "Importância do NutriSUS" e as "Escolas como ambiente promotor de saúde". Resultados: Os pais demonstraram algum conhecimento sobre a importância/ benefício do NutriSUS, reconhecido pelo aumento do apetite dos seus filhos e o entendimento da oferta de uma alimentação mais enriquecida nutricionalmente. Eles ainda apresentaram uma percepção positiva da estratégia de parceria entre escola e unidade de saúde e a escola como ambiente promotor de saúde. Conclusão: O relato dos pais indicou que eles compreendem o objetivo da estratégia de fortificação e a importância de uma alimentação saudável nos primeiros anos de vida. Esse conhecimento contribui para melhorar o suporte ao crescimento e desenvolvimento das crianças brasileiras uma vez que os pais são agentes condicionantes para a efetividade dessa estratégia.

Descritores: Alimentação Infantil; Micronutrientes; Saúde Pública.

\section{ABSTRACT}

Objective: To assess parents' perception of the implementation of the strategy for the fortification of child food with micronutrient powders (NutriSUS). Methods: Qualitative descriptive and exploratory study conducted from August to September 2015 with 11 mothers of children enrolled in preschool programs in Montes Claros, Minas Gerais, Brazil. Data were collected using a semi-structured questionnaire and then underwent content analysis from which two thematic categories emerged: "Importance of NutriSUS" and "Schools as healthpromoting environments". Results: Parents showed to be aware of the importance/benefits of NutriSUS given the increase in child's appetite and provision of a nutritionally enriched diet. They also had a positive perception of the strategy of partnership between the school and the health center and of the school as a health-promoting environment. Conclusion: Parents' reports indicated that they understood the objective of the fortification strategy and the importance of a healthy diet in the first years of life. This knowledge contributes to improve the support for the growth and development of Brazilian children given that parents are important agents for the effectiveness of the strategy.

Descriptors: Child Nutrition; Micronutrients; Public Health.

\author{
Rodrigo Pereira Prates ${ }^{(1)}$ \\ Mariana Mendes Pereira ${ }^{(1)}$ \\ Lucinéia de Pinho ${ }^{(2,3)}$
}

1) Faculdade de Saúde Ibituruna - FASI Montes Claros (MG) - Brasil

2) Faculdades Unidas do Norte de Minas FUNORTE - Montes Claros (MG) - Brasil

3) Universidade Estadual de Montes Claros - UNIMONTES - Montes Claros (MG) -

Brasil

Recebido em: 06/04/2016

Revisado em: 14/06/2016 Aceito em: 22/08/2016 


\section{RESUMEN}

Objetivo: Analizar la percepción de los padres de los alumnos sobre la implantación de la estrategia de fortificación de la alimentación infantil con micronutrientes en polvo (NutriSUS). Métodos: Investigación cualitativa, descriptiva y exploratoria realizada en los meses de agosto y septiembre de 2015 con 11 madres de alumnos matriculados en el Centro de Educación Infantil de la ciudad de Montes Claros, MG, Brasil. Los datos fueron recogidos a través de una entrevista semiestructurada y analizados a través de la técnica de análisis de contenido con la identificación de dos categorías temáticas "La Importancia del NutriSUS" y "Las escuelas como ambiente de promoción de la salud”. Resultados: Los padres demuestran algún conocimiento sobre la importancialel beneficio del NutriSUS reconocido por el aumento del apetito de sus hijos y el entendimiento de la oferta de una alimentación más rica de nutrientes. Ellos presentaron una percepción positiva de la estrategia de acuerdo entre la escuela y la unidad de salud y la escuela como ambiente de promoción de la salud. Conclusión: El relato de los padres indicó su comprensión del objetivo de la estrategia de fortificación y la importancia de una alimentación saludable en los primeros años de vida. Ese conocimiento contribuye para mejorar el apoyo al crecimiento $y$ desarrollo de niños brasileños ya que los padres son factores condicionantes para la efectividad de esa estrategia.

Descriptores: Nutrición del Niño; Micronutrientes; Salud Pública.

\section{INTRODUÇÃO}

A nutrição adequada nos primeiros anos de vida da criança é básica para o desenvolvimento saudável da criança ${ }^{(1)} \mathrm{e}$, quando inadequada, pode trazer transtornos como a carência de nutrientes, o desenvolvimento precoce de sobrepeso e obesidade e doenças crônicas associadas ${ }^{(2)}$. Por outro lado, crianças submetidas a práticas alimentares ideais alcançam seu desenvolvimento normal e se tornam adultos mais saudáveis, com maior capacidade intelectual e produtiva ${ }^{(1)}$.

Dados epidemiológicos mostram a gravidade da desnutrição no Brasil. Em um estudo realizado em 1.214 crianças menores de cinco anos no Maranhão, reportou-se desnutrição em 4,5\% pelo critério peso para idade, $3,9 \%$ pelo critério peso para estatura e $8,5 \%$ considerandose baixa estatura para idade ${ }^{(3)}$. No Acre, de 667 crianças analisadas, 9,9\% apresentavam déficit de estatura para idade e $4,1 \%$ déficit de peso para estatura ${ }^{(4)}$. No município de Serro, Minas Gerais, de 722 crianças de 2 a 5 anos avaliadas, $10,66 \%$ encontravam-se desnutridas ${ }^{(5)}$.

Devido ao crescimento rápido e imaturidade fisiológica e imunológica, as crianças representam um grupo de grande vulnerabilidade à ingestão adequada de micronutrientes ${ }^{(1)}$.
Essa é uma questão que merece atenção visto que a carência de micronutrientes aumenta a suscetibilidade das crianças a diarreias e infecções e pode comprometer a maturação do sistema nervoso, visual, mental e intelectual ${ }^{(6)}$.

As carências infantis de micronutrientes são consideradas um real problema de saúde pública mundial, sobretudo nos países em desenvolvimento, onde abrangem altas $\operatorname{taxas}^{(7)}$. No Brasil, as carências de micronutrientes mais observadas são as deficiências de ferro e vitamina $\mathrm{A}^{(8)}$. Em 2006, de acordo com dados da Pesquisa Nacional de Demografia e Saúde (PNDS), 20,9\% das crianças brasileiras menores de cinco anos tinham anemia ${ }^{(9)}$, o que se associa à falta de ferro.

A anemia ferropriva, isto é, decorrente da deficiência de ferro no organismo, é a mais comum das doenças causadas por carência nutricional e afeta mais da metade da população mundial ${ }^{(10)}$, especialmente os grupos de lactantes e pré-escolares. No Brasil, estudos realizados em diferentes cenários epidemiológicos demonstraram que $52 \%$ das crianças acompanhadas em creches possuíam essa patologia $^{(11)}$. Apenas em pré-escolares de creches, um estudo em Belo Horizonte, MG-BR, demonstrou prevalência de anemia em $37,3 \%$ das crianças $^{(11)}$ e outro realizado em Vitória, ES, BR, encontrou essa doença em $37 \%$ delas $^{(12)}$.

As causas mais comuns de anemia incluem o baixo consumo e ou a baixa absorção do ferro dietético ${ }^{(13)}$. Em crianças, a anemia é comumente associada à interrupção precoce do aleitamento materno exclusivo e ingestão de substâncias como antiácidos, fitatos, fosfatos, oxalatos e taninos, as quais diminuem a absorção de ferro ${ }^{(14)}$. Os efeitos adversos da anemia são graves, repercutindo sobre a capacidade cognitiva, comportamento, crescimento e imunidade celular ${ }^{(11)}$.

No intuito de prevenir doenças causadas por carência nutricionais, o Ministério da Saúde juntamente com o Ministério do Desenvolvimento Social e Combate à Fome e Ministério da Educação lançou, no segundo semestre de 2014, o NutriSUS, que é uma estratégia de fortificação da alimentação infantil com micronutrientes em pó(15). Essa estratégia tem como público alvo crianças de seis meses a 48 meses de idade, matriculadas em creches integrantes do Programa Saúde na Escola. A estratégia consiste na distribuição de sachês de $1 \mathrm{~g}$ cada que, contendo uma combinação de 15 micronutrientes em pó, devem ser adicionados em uma das refeições da criança no momento em que ela for comer ${ }^{(15)}$.

As recomendações do uso de sachês em escolas ou creches devem observar as normas da Resolução da Diretoria Colegiada (RDC) $n^{\circ}$ 316/2014 da Agência Nacional de Vigilância Sanitária (ANVISA), pois mesmo se tratando de um alimento em pó, deve seguir as normas de 
higiene do preparo até o consumo ${ }^{(16)}$. O conteúdo do sachê deve ser adicionado a uma das refeições, misturado a uma pequena porção da comida que pode ter consistência pastosa ou mesmo no arroz e feijão. Deve-se evitar aquecer o pó ou adicioná-lo a líquidos ou alimentos duros para não haver perda de nutrientes. A mistura deve ser feita em refeições individuais, e coletivas, e as crianças que fazem parte da suplementação do NutriSUS não necessitam receber outra tipo de suplementação de ferro ${ }^{(15)}$. Já foi demonstrado que, no período de um ano, a estratégia de fortificação com micronutrientes reduz a deficiência de ferro em $51 \%$, e a prevalência de anemia em $31 \% \%^{(17)}$.

O sucesso de programas como o NutriSUS depende em grande parte dos pais, pois a suplementação alimentar deve ser inserida no cotidiano das crianças. Os hábitos alimentares se formam na infância ${ }^{(18)}$ e nesse processo os pais têm um papel fundamental ao adotarem muito cedo um ambiente alimentar familiar, o contexto alimentar da criança. As atitudes, crenças e práticas alimentares dos pais, adaptam-se as ofertas de alimentos, influenciam sobre o tempo, quantidade e contexto social que envolve as refeições, para além de colocarem o ambiente emocional que as envolve $\mathrm{e}^{(18,19)}$.

Tendo em vista que os pais atuam como agentes que propiciam a efetividade de estratégias como essa. Questiona-se, o que os pais compreendem sobre a implantação da estratégia de fortificação da alimentação infantil com micronutrientes em pó (NutriSUS)?

O presente estudo tem como objetivo analisar a percepção dos pais dos educandos acerca da implantação da estratégia de fortificação da alimentação infantil com micronutrientes em pó (NutriSUS).

\section{MÉTODOS}

Trata-se de um estudo qualitativo, de caráter descritivo e exploratório, realizado nos meses de agosto e setembro de 2015, em um Centro Municipal de Educação Infantil (CEMEI) localizado em região periférica da cidade de Montes Claros, MG- Brasil, contemplado com a estratégia do NutriSUS,

Participaram deste estudo mães de educandos matriculados no CEMEI e que estavam recebendo a suplementação no momento da pesquisa. A amostra foi considerada como satisfatória, apesar das informações dos analisados tornaram-se repetitivas, deixando de acrescentar novas informações ${ }^{(20)}$.

Os critérios de inclusão no estudo incluíram participação das mães na reunião de conscientização do NutriSUS no CEMEI, assinatura do termo de consentimento permitindo a participação dos seus filhos na estratégia NutriSUS, adesão da criança à suplementação desde o início da administração do suplemento e ter disponibilidade de tempo para responder às perguntas. Foram excluídos os participantes que não acompanharam o programa NutriSUS no CEMEI, desde a implantação até o momento da coleta das informações, e as mães das crianças que não estavam consumindo a suplementação regularmente. Assim, fizeram parte do estudo 11 mães das crianças matriculadas no CEMEI.

$\mathrm{O}$ instrumento de coleta de dados foi um roteiro semiestruturado. As entrevistas foram individuais $\mathrm{e}$ ocorreram em dois blocos, sendo o primeiro relacionado à caracterização da população com as variáveis: Idade, estado civil, grau de escolaridade. No segundo bloco, tratou-se da questão norteadora do estudo: "Quais os benefícios que o NutriSUS trouxe para o seu filho(a)/comunidade?" A coleta dos dados aconteceu após ao término das aulas, no momento em que os pais buscavam os seus filhos no CEMEI.

As entrevistas duravam cerca de 20 minutos, foram gravadas e transcritas para avalizar a inteireza das declarações e requerer a fidedignidade dos dados. Para a análise dos dados, empregou-se a técnica de análise do conteúdo $^{(21)}$, na modalidade temática, sem definição prévia das categorias. Primeiramente, obteve-se a leitura flutuante das entrevistas, deliberando as unidades de registro e de contexto. Em seguida, determinaram-se as categorias de análise, agrupando as unidades de registro e contexto com os atributos similares ou que se relacionavam entre si, sendo identificadas as categorias: "Importância do NutriSUS" e as "Escolas como ambiente promotor de saúde". Por fim, realizou-se à interpretação dos dados, buscando encontrar o conteúdo subjacente ao que foi manifestado. $\mathrm{Na}$ análise dos resultados, as falas foram organizadas por códigos de $\mathrm{M}$ para mãe, sendo cognominadas por M1 a M11 para garantir $\mathrm{o}$ anonimato.

O estudo foi aprovado pelo Comitê de Ética e Pesquisa da Universidade Estadual de Montes Claros (UNIMONTES), sob parecer n ${ }^{\circ} .911 .383$, de 11 de dezembro de 2014, seguindo, dessa forma, os princípios norteadores de pesquisa com seres humanos, conforme Resolução $\mathrm{n}^{\circ}$. 466/12 do Conselho Nacional de Saúde.

\section{RESULTADOS E DISCUSSÃO}

A seguir serão apresentados os dados de identificação das mães entrevistadas. Após definir as duas categorias temáticas, os resultados serão analisados dentro de cada uma, conforme exposto.

\section{Dados de identificação das mães entrevistadas}

As 11 mães entrevistadas tinham entre 18 e 46 anos de idade e mostraram envolvimento no cuidado com os filhos. 
A maioria vivia em união estável, duas eram separadas dos companheiros e todas apresentavam baixo nível socioeconômico, o que reforça a importância do auxílio do governo para promoção da alimentação saudável por meio da fortificação da dieta com micronutrientes. No que se refere ao nível de instrução, nove mães haviam cursado o ensino médio completo e apenas duas tinham ensino médio incompleto.

\section{Importância do NutriSUS}

Essa categoria trata da percepção dos pais acerca da importância e benefícios do NutriSUS. Seus depoimentos indicam entendimento de que a suplementação promove enriquecimento nutricional da alimentação, e que as vitaminas contidas nela trazem benefícios ao crescimento e fortalecem a criança, que desenvolve maior resistência às doenças. Houve ainda relato de respostas positivas das crianças à fortificação, especialmente em relação ao aumento de apetite e melhoria na disposição da criança. Essas impressões são exemplificadas a seguir, em trechos de algumas entrevistas:

“[...] ele está recebendo uma alimentação saudável e o suplemento ajudaria a não ter anemia e outras doenças né e até porque meu filho não amamentou então é uma ajuda que ele tem"(P11).

"[...]como ele está recebendo essas vitaminas isso vai melhorar a alimentação e pode auxiliar no crescimento dele" (P1).

"[...]acho que o principal beneficio é no aumento do apetite, combatendo a desnutrição ou deficiência de alguma vitamina ou mineral" (P2).

O objetivo principal da estratégia de fortalecimento de dieta com microminerais é reforçar o desenvolvimento infantil e a prevenção de deficiências de vitaminas e minerais, especialmente da anemia e deficiência de ferro $^{(22)}$. Os benefícios da suplementação na primeira infância estão ligados principalmente ao estágio crítico de desenvolvimento nessa fase. Estudos associam o uso da estratégia com a redução de $50 \%$ do risco de anemia e 79\% do risco de carência de ferro, além de melhorar os índices antropométricos e o desempenho cognitivo das crianças ${ }^{(22)}$. No entanto, por melhor que se estruture a NutriSUS, o suporte e colaboração da família para o sucesso do resultado é indispensável. Daí a necessidade de se avaliar as impressões parentais acerca do assunto.

Estudos relacionados à nutrição infantil enfatizam a importância de se conhecer a impressão dos pais ${ }^{(23,24)}$ porque, dentre os múltiplos fatores associados ao estado de saúde da criança, constam aspectos culturais impregnados na família ${ }^{(25)}$. Assim, as atitudes e práticas alimentares dos pais modelam a oferta de alimentos dos filhos, assim como quantidade e qualidade das refeições. Os estudos em países desenvolvidos têm mostrado exemplos dessa associação, por exemplo, crianças cujos pais apresentam sobrepeso ou obesidade têm maior chance de também o terem ${ }^{(26)}$.

$\mathrm{O}$ esclarecimento dos pais e integrações deles em programas como a estratégia NutriSUS tem ainda o papel de chamar sua atenção para a possibilidade de seus filhos sofrerem carências nutricionais e desenvolverem doenças relacionadas, como a anemia. A percepção parenteral do estado nutricional da criança é um aspecto que tem sido investigado porque uma percepção alterada ou o não reconhecimento pelos pais do estado nutricional distrófico dos seus filhos pode comprometer a adoção de medidas preventivas ou de tratamento perante essa situação ${ }^{(18)}$.

\section{Escola como ambiente promotor de saúde}

Nessa categoria são apresentadas as percepções dos pais sobre a escola como parceria da unidade de saúde e, portanto, ambiente promotor da saúde da criança. A articulação entre os setores públicos de saúde e educação é preconizada pela Organização Mundial de Saúde para a adoção de medidas integrantes que diminuam a anemia no país ${ }^{(15)}$. É o caso da ação contígua entre escola e unidade de saúde na prática de estratégias de fortificação da alimentação.

No presente estudo, observou-se que a parceria entre escola e unidade de saúde para a promoção da estratégia NutriSUS foi bem assimilada pelas mães entrevistadas. Elas ressaltaram que, através dessa parceria, seus filhos tinham acesso a uma alimentação mais saudável e, consequentemente, melhoria na saúde, conforme as falas a seguir:

"[...]é a partir dessa iniciativa entre escola e o posto que as crianças estão comendo melhor para crescer com saúde e ajudar a não pegar doenças" (P4).

"[...] Sim pelo fato que o posto de saúde não tem muito contato igual aqui na escola eles tão tendo, porque a gente só procura o posto quando a gente tá precisando de alguma coisa e caso a criança adoece ai a gente procura e na escola eles já estão tendo esse acompanhamento. E o meu filho passa muito tempo aqui na escola e ele se alimenta na escola então ajuda bastante" (P11).

As mães ressaltaram inclusive que as crianças que recebem o suplemento nas escolas estão menos vulneráveis às doenças, evitando a necessidade de levá-las ao posto de saúde. A diminuição à procura pelos centros de saúde pode contribuir para desafogar o atendimento nos postos de saúde e refletir em melhoria no serviço prestado. Essas impressões são descritas a seguir:

"[...]é uma parceria da escola, do posto e da família, envolvendo todo mundo, a gente traz as crianças para a 
escola porque a gente confia na escola e recebendo essas vitaminas aqui só tende a melhorar a saúde das crianças e não precisa ficar levando as crianças no posto direto" (P6).

"[...]facilita para gente que trabalha e não pode ficar com os meninos o dia todo, eles vêm para escola, aprendem e ainda cuidam da saúde deles." (P8).

A ESF sugere que esse intercâmbio escola /unidade de saúde vá além do corpo biológico e alcance os seres humanos em sua complexidade e integralidade ${ }^{(27)}$. Portanto, carecem de introduzir ações que extrapolem os muros das unidades de saúde e alcancem o contexto histórico, social, cultural, político e econômico dos indivíduos ou coletivos, no sentido de promover a saúde das pessoas. A complexidade dessa estratégia evidencia a inabilidade do setor saúde de operar isoladamente, sendo imperativo fazer parcerias entre distintos segmentos sociais, dentre eles, o setor educação, representado pelas escolas ${ }^{(28)}$.

As ações de promoção e de prevenção da saúde são realizadas preferencialmente de forma intersetorial. Nesse contexto, destaca-se a escola como um ambiente importante para o desenvolvimento de ações preventivas com enfoque para a educação em saúde ${ }^{(29)}$. Acredita-se que esses ambientes sejam propícios para a execução de ações coletivas e se configurem numa oportunidade de reforço de ações que contribuam para o pleno desenvolvimento infantil. Além disso, há de se considerar a realidade de que muitas crianças matriculadas em escolas públicas de educação infantil no Brasil fazem suas principais refeições na escola, configurando importante oportunidade de desenvolvimento da ação e aumentando o alcance da estratégia de prevenção de anemia e outras carências nutricionais ${ }^{(15)}$. É no ambiente escolar que a equipe de saúde consegue uma maior interação com crianças para a realização de atividades educativas que podem conscientizá-las e torná-las corresponsáveis pela sua saúde ${ }^{(30)}$.

No contexto do presente estudo, a fortificação da alimentação com micronutrientes pode ser feita em qualquer lugar onde a criança faz suas refeições, pode ser na sua casa, nas creches, escolas ou qualquer outro lugar próprio para a ação ${ }^{(31)}$. Porém, considerando a alimentação saudável como um conteúdo educativo, ao longo dos anos, pais e educadores vêm concordando com a necessidade da escola assumir um papel protagonista nesse trabalho ${ }^{(32)}$ e formular mecanismos para inserir o grupo familiar no processo de construção coletiva da aprendizagem do aluno, fortalecendo o binômio pais-escolas ${ }^{(33)}$. Os pais dos educandos reconheceram a importância da parceria e da escola como ambiente adequado para o desenvolvimento da estratégia, como representado na seguinte fala: "[...]a iniciativa é muito boa, já que onde eu moro é muito longe do ESF, assim quando junta o ESF com a escola facilita para nós pais que às vezes não temos tempo pra levar nossos filhos até lá. Dessa forma permite com que mais crianças participem do programa, até as mais carentes que não têm dinheiro para comprar suplementação ou não têm condições de se locomover para grandes distâncias" (P2).

Os pais entendem as escolas como um importante ambiente promotor da saúde porque é onde os estudantes permanecem grande parte do dia e podem ter acesso a uma alimentação balanceada e saudável. Isso é particularmente benéfico frente ao baixo poder aquisitivo e a falta de acesso à informação pelas famílias assistidas pela estratégia de fortificação alimentar. Os pais enfatizaram a importância da implantação do programa no ambiente escolar, como exemplificado nas seguintes falas:

"[...] essa atitude em conjunto auxilia na melhora da saúde de uma forma geral, fazendo com que aconteça um programa que previna e trate doenças, evitando a sobrecarga de procura nos ESF e hospitais, pois as crianças adoecem menos.” (P5).

"[...]pois eles passam muito tempo na escola, então aqui é um ótimo lugar para eles se alimentarem bem, até porque muitas vezes em casa a gente não tem condições de dar toda a alimentação que eles precisam" (P7).

Há estudos que reportam a grande prevalência de pais e responsáveis pelas crianças que não seguem as orientações dos profissionais de saúde em relação à alimentação, de modo que as estratégias realizadas em parceira da escola com as unidades de saúde auxiliam na diminuição das consequências negativas dessa não adesão ${ }^{(34,35)}$. É por isso que é nesse ambiente que políticas públicas de saúde direcionadas para a alimentação e a nutrição estão sendo colocadas em prática ${ }^{(36)}$.

Os relatos mostram que a escola representa do ponto de vista dos pais um ambiente de destaque para a realização de programas de educação alimentar e nutricional, propiciando a formação de novos hábitos ainda na idade infantil, quando suas experiências extrapolam o convívio familiar. Isso vai de acordo com outros estudos que reportam que, pela ótica dos pais, as refeições ofertadas às crianças na creche são mais adequadas que as oferecidas em casa ${ }^{(36)}$. Eles ainda enfatizam a necessidade da comunicação entre creche e família a fim de fortalecer o pleno desenvolvimento das crianças $^{(37)}$. Entretanto, há um entendimento dos pais de que a construção de hábitos alimentares saudáveis é importante e não deve ser atribuída apenas ao ambiente escolar, mas que deve ser promovida pela ação conjunta de programas governamentais vigentes, creches e família ${ }^{(38,39)}$. 


\section{CONSIDERAÇÕES FINAIS}

O presente estudo contribui para promoção da saúde infantil por buscar identificar a percepção dos agentes que influenciam diretamente a formação dos costumes alimentares das crianças, especialmente aquelas que convivem boa parte do dia longe dos familiares, ou seja, em creches públicas. A partir da análise da percepção dos pais dos educandos, pôde-se identificar um parecer positivo com relação à estratégia de fortificação da alimentação dos seus filhos no que tange o fortalecimento da saúde das crianças e a prevenção de doenças por meio da fortificação da alimentação.

Além disso, foi possível identificar o impacto positivo dessa estratégia na comunidade como um todo através da parceria entre escola e ESF para a promoção da saúde. Essa intersetorialidade configura-se como uma prática eminente e necessária para que ações de saúde atinjam os públicos mais remotos e carentes, inserindo a percepção da corresponsabilidade pela sua própria saúde e pela saúde de sua família. O conceito e adoção da corresponsabilidade em saúde são essenciais para a emancipação e autoafirmação em atitudes individuais de cuidados à saúde. Isso, entretanto, não se verifica na realidade em função das condições de vida desfavoráveis e o pouco conhecimento em saúde que goza esse público.

Num contexto interdisciplinar, deve-se também considerar que a responsabilidade pela alimentação infantil, particularmente a ingestão adequada de nutrientes que sustente o pleno crescimento e desenvolvimento da criança, deve ser compartilhada entre diferentes grupos sociais, incluindo, além do núcleo familiar, centros de educação infantil onde as crianças passam grande parte do dia e desenvolvem suas atividades diárias, assim como os centros de ESF e os governos das esferas municipal, estadual e federal. Enfim, todos aqueles que direta ou indiretamente estejam envolvidos no cuidado infantil.

Dessa forma, é fundamental que além de elaborar projetos de intervenção em saúde para essa população, seja analisada a avaliação da percepção dos agentes condicionadores da efetividade desses programas, como proposto no presente estudo.

\section{REFERÊNCIAS}

1. Carvalho CA, Fonsêca PC, Priore SE, Franceschini SC, Novaes JF. Consumo alimentar e adequação nutricional em crianças brasileiras: revisão sistemática. Rev Paul Pediatr. 2015;33(2):211-21.

2. S Filha EO, Araújo JS, Barbosa JS, Gaujac DP, Santos CFDS, et al. Consumption of food groups among children attending the public health system of Aracaju, Northeast Brazil, in Sergipe. Rev Paul Pediatr. 2012; 30(4): 529-36.

3. Chagas DC, Silva AAM, Batista RFL, Simões VMF, Lamy ZC, Coimbra LC, et al. Prevalência e fatores associados à desnutrição e ao excesso de peso em menores de cinco anos nos seis maiores municípios do Maranhão. Rev Bras Epidemiol. 2013;16(1):146-56.

4. Souza OF, Benício MHD, Castro TGM, Muniz PT, Cardoso MA. Desnutrição em crianças menores de 60 meses em dois municípios no Estado do Acre: prevalência e fatores associados. Rev Bras Epidemiol. 2012;15(1):211-21.

5. Vargas AM, Abreu AC, Machado BL, Rodrigues ECA, Neves NN, Moura MDG, et al. Perfil nutricional em crianças de 2 a 5 anos no Município de Serro, Minas Gerais. Rev Univ Vale do Rio Verde. 2015;13(1): 305-14.

6. Bernardi JR, De Cezaro C, Fisberg RM, Fisberg M, Rodrigues GP, Vitolo MR. Dietary micronutrient intake of preschool children at home and in kindergartens of the municipality of Caxias do Sul (RS), Brazil. Rev Nutr. 2011;24(2):253-61.

7. World Health Organization, World Food Programme, UNICEF. Preventing and controlling micronutrient deficiencies in populations affected by an emergency: multiple vitamin and mineral supplements for pregnant and lactating women, and for children aged 6 to 59 months [Internet]. Geneva: WHO; 2007. [acesso em 2015 Nov 20]. Disponível em: http:// www.who.int/nutrition/publications/WHO_WFP UNICEFstatement.pdf

8. Ministério da Saúde (BR), Secretaria de Atenção à Saúde, Departamento de Atenção Básica. Programa Nacional de Suplementação de Ferro: manual de condutas gerais [Internet]. Brasília: Ministério de Saúde; 2013 [acesso em 2015 Nov 10]. Disponível em: http://bvsms.saude.gov.br/bvs/publicacoes/manual_ suplementacao_ferro_condutas_gerais.pdf

9. Ministério da Saúde (BR). Centro Brasileiro de Análise e Planejamento. PNDS 2006: dimensões do processo reprodutivo e da saúde da criança [acesso em 2015 Nov 10]. Disponível em: http://bvsms.saude.gov.br/bvs/ publicacoes/pnds_crianca_mulher.pdf

10. World Health Organization. Infant and young child feeding: model chapter for textbooks for medical students and allied health professionals. Geneva: WHO; 2009. 
11. Oliveira TDSC, Silva MC, Santos JN, Silva-Rocha D, Alves CRL et al. Anemia entre pré-escolaresum problema de saúde pública em Belo Horizonte, Brasil. Ciênc Saúde Coletiva. 2014;19(1):59-66.

12. Oliveira APDND, Pascoal MN, Santos LCD, Pereira SCL, Justino LEH, Petarli GB, et al. Prevalência de anemia e sua associação com aspectos sociodemográficos e antropométricos em crianças de Vitória, Espírito Santo, Brasil. Ciênc Saúde Coletiva. 2013;18(11):3273-80.

13. Coutinho GGPL, Cury PM, Cordeiro JA. Cyclical iron supplementation to reduce anemia among Brazilian preschoolers: a randomized controlled trial. BMC Public Health. 2013;13(1):21.

14. Silva EBD, Villani MS, Jahn ADC, Cocco M. . Fatores de risco associados à anemia ferropriva em crianças de 0 a 5 anos, em um município da região noroeste do Rio Grande do Sul. REME Rev Min Enferm. 2011;15(2):165-73.

15. Ministério da Saúde (BR), Secretaria de Atenção à Saúde, Departamento de Atenção Básica. NutriSUS Caderno de Orientações Operacionalização da estratégia de fortificação da alimentação infantil com micronutrientes (vitaminas e minerais) em pó [Internet]. Brasília: Ministério da Saúde; 2015 [acesso em 2015 Dez 11]. Disponível em: http://189.28.128.100/dab/ docs/portaldab/publicacoes/caderno_orientacoes_ nutrisus.pdf

16. Ministério da Saúde (BR). Agência Nacional de Vigilância Sanitária Resolução da Diretoria Colegiada - RDC n 14, de 28 de março de 2014 [acesso em 2015 Dez 11]. Disponível em: http://portal.anvisa.gov.br/ documents/33880/2568070/rdc0014_28_03_2014. pdf/9a5267c3-848f-4c62-b305-e63f25d6118 e

17. De-Regil, Suchdev PS, Vist GE, Walleser S, PeñaRosas JP. Home fortification of foods with multiple micronutrient powders for health and nutrition in children under two years of age. Cochrane Database Syst Rev. 2011;7(9):CD008959.

18. Aparício G, Cunha M, Duarte J, Pereira A. Olhar dos Pais sobre o Estado Nutricional das Crianças Préescolares. Millenium. 2011;40:99-113.

19. Flores-Peña Y, Ortiz-Félix RE, Cárdenas-Villarreal VM, Ávila-Alpirez H, Alba-Alba CM, HernándezCarranco RG. Estratégias maternas referentes à alimentação e à atividade física e sua relação com o estado nutricional dos filhos. Rev Latinoam Enferm. 2014;22(2):286-92.
20. Minayo MCS. O desafio do conhecimento: pesquisa qualitativa em saúde. São Paulo: Hucitec; 2007.

21. Bardin L. Análise de conteúdo. Lisboa: Edições 70; 1979.

22. Low M, Farrell A, Biggs BA, Pasricha SR. Effects of daily iron supplementation in primary-schoolaged children: systematic review and meta-analysis of randomized controlled trials. Can Med Assoc J. 2013;185(17):E791-E802.

23. Fechine ÁD, Machado MMT, Lindsay AC, Fechine VAL, Arruda CAM. Percepção de pais e professores sobre a influência dos alimentos industrializados na saúde infantil. Rev Bras Promoç Saúde. 2015;28(1): $16-22$.

24. Frota MA, Bezerra JA, Férrer MLS, Martins MC, Silveira VG. Percepção materna em relação ao cuidado e desenvolvimento infantil. Rev Bras Promoç Saúde. 2011;24(3):245-50.

25. Murray R, Bhatia J, Okamoto J, Allison M, Ancona R, Attisha E et al. Snacks, sweetened beverages, added sugars, and schools. Pediatrics. 2015;135(3):575-83.

26. Dalcastagné G, Ranucci JMA, Nascimento MA, Liberali R. Influência dos pais no estilo de vida dos filhos e sua relação com a obesidade infantil. RBONE. 2012;2(7): 5 .

27. Silva KL, Rodrigues AT. Ações intersetoriais para promoção da saúde na Estratégia Saúde da Família: experiências, desafios e possibilidades. Rev Bras Enferm. 2010;63(5):762-9.

28. Souza MF. O Programa Saúde da Família no Brasil: análise do acesso à atenção básica. Rev Bras Enferm. 2008; 61(2):153-8.

29. Andrade TM, Melo GC, Santos SMP, Martins VM, Policarpo MDFO. Educação em Saúde: uma experiência de integração entre a equipe de saúde da família e a equipe do PET Saúde. Rev EXPOPEP. 2014;1(1):1-9.

30. Maciel ELN, Oliveira CB, Frechiani JM, Sales CMM, Brotto LDDA, Araújo MD. Projeto Aprendendo Saúde na Escola: a experiência de repercussões positivas na qualidade de vida e determinantes da saúde de membros de uma comunidade escolar em Vitória, Espírito Santo. Ciênc Saúde Coletiva. 2010;15(2): 389-96.

31. World Health Organization. Use of multiple micronutrient powders for home fortification of foods consumed by infants and children 6-23 months 
of age. Geneva: WHO; 2011 [acesso em 2015 Nov 18]. Disponível em: http://www.who.int/elena/titles/ guidance_summaries/micronutrientpowder_infants/en/

32. Gonçalves FD, Catrib AMF, Vieira NFC, Vieira LJEDS. A promoção da saúde na educação infantil. Interface Comun Saude Educ. 2008;12(24):181-92.

33. Souza MMA, Enumo SRF, Paula KMP, Souza RV, Bezerra RS, Mendes KB. Promoção de comportamentos saudáveis em pré-escolares. Rev Bras Promoç Saúde. 2013;26(3):387-95.

34. Broilo MC, Louzada MLC, Drachler ML, Stenzel LM, Vitolo MR. Percepção e atitudes maternas em relação às orientações de profissionais de saúde referentes a práticas alimentares no primeiro ano de vida. J Pediatr (Rio J). 2013;89(5):485-91.

35. Campos AAO, Cotta RMM, Oliveira JM, Santos AK, Araújo RMA. Nutritional counseling for children under two years of age: opportunities and obstacles as strategic challenges. Ciênc Saúde Coletiva. 2014;19(2):529-38.

36. Ramos FP, Santos LAS, Reis ABC. Educação alimentar e nutricional em escolares: uma revisão de literatura. Cad Saúde Pública. 2013;29(11):2147-61.
37. Afonso FM, Scofano VB, Silva ER, Oliveira DM, Silva CC, Rocha FHZ, et al. Percepção dos responsáveis sobre a alimentação de crianças menores de 4 anos creches públicas-RJ [abstract]. In: $11^{\circ}$ Congresso Internacional da Rede Unida; 2014 Apr 10-13; Botucatu: Interface; 2014. p. 1.

38. Fechine ADL, Machado MMT, Lindsay AC, Fechine VAL, Arruda CAM. Percepção de pais e professores sobre a influência dos alimentos industrializados na saúde infantil. Rev Bras Promoç Saúde. 2015;28(1): 16-22.

39. Camargo APPM; Barros-Filho AA, Antonio MARGM, Giglio JS. A não percepção da obesidade pode ser um obstáculo no papel das mães de cuidar de seus filhos. Ciênc Saúde Coletiva. 2013;18(2):323-33.

\section{Endereço para correspondência:}

Rodrigo Pereira Prates

Faculdade de Saúde Ibituruna - FASI

Avenida Nice, 99

Bairro: Ibituruna

CEP: 39401-328 - Montes Claros - MG - Brasil

E-mail: rodrigo_pprates@yahoo.com.br 